\title{
A New Transport Regime in the Quantum Hall Effect
}

\author{
D. Shahar, M. Hilke, C.C. Li and D. C. Tsui \\ Department of Electrical Engineering, Princeton University, Princeton New Jersey, 08544 \\ S. L. Sondhi \\ Department of Physics, Princeton University, Princeton New Jersey, 08544 \\ M. Razeghi \\ Department of Electrical Engineering, Northwestern University, Evanston Illinois, 60208
}

(October 4, 2018)

Our evolving understanding of the dramatic features of charge-transport in the quantum Hall (QH) regime has its roots in the more general problem of the metal-insulator transition. Conversely, the set of conductivity transitions observed in the QH regime provides a fertile experimental ground for studying many aspects of the metal-insulator transition. While earlier works [1,2] tend to concentrate on transitions between adjacent QH liquid states, more recent works [3] focus on the transition from the last $\mathrm{QH}$ state to the high-magnetic-field insulator. Here we report on measurements that identified a novel transport regime which is distinct from both, the fully developed QH liquid, and the critical scaling regime believed to exist asymptotically close to the transition at very low temperatures ( $T$ 's). This new regime appears to hold in a wide variety of samples and over a large range of magnetic field $(B)$ and temperature. It is characterized by a remarkably simple phenomenological scaling of the longitudinal resistivity $\left(\rho_{x x}\right)$, which is the center of this letter, and is not understood theoretically.

We begin by focusing on a recent set of observations that directly begot some of the results presented here. In Refs. [9, 10], the observation of a new symmetry was reported, relating the transport properties of the QH liquid to those of the adjacent insulator. For the case of the $\nu=1$ to insulator transition, this symmetry is summarized by:

$$
\rho_{x x}(\Delta \nu)=1 / \rho_{x x}(-\Delta \nu)
$$

where $\nu$ is the Landau level filling factor, $\Delta \nu=\nu-\nu_{c}$ and $\nu_{c}$ is the critical $\nu$ of the transition (see the inset of Fig. 1 for the identification of $\nu_{c}$ ). Remarkably, a similar symmetry holds at transitions from the $1 / 3$ fractional quantum Hall $(\mathrm{FQH})$ state to the insulator, if one replaces the $\nu$ 's in Eq. 1 with those of composite fermions [11]. In addition, in ref. [9] we showed that a generalized relation holds, within experimental error, even for the non-linear regime of transport, and suggested the possibility that duality symmetry underlies this relation [9,12]. More recently, a similar relation was observed in Si-MOSFET samples near the $B=0$ conductor-insulator transition [13], which raises the question whether a more general explanation may exist for the symmetry 14.

In the remainder of this paper we shall present and discuss a view of the $\rho_{x x}$ data where the symmetry of Eq. 1 is a straightforward ingredient. We begin by plotting, in Fig. $1, \rho_{x x}$ vs. $\nu$ for a low mobility $(\mu=30000$ $\left.\mathrm{cm}^{2} / \mathrm{Vsec}\right)$, low density $\left(n=3 \cdot 10^{10} \mathrm{~cm}^{-2}\right)$, InGaAs/InP sample, in the range of $0.4<\nu<0.8$ which includes the $\nu=1$-to-insulator transition $(\nu=0.562)$, at several $T$ 's between 0.072 and $2.21 \mathrm{~K}$. Rather than plotting the data using the conventional linear ordinate (see inset of Fig. 1 ), we chose a log scale, which clearly reveals a distinct $\nu$ dependence of $\rho_{x x}$ :

$$
\rho_{x x}=e^{\frac{-\Delta \nu}{\nu_{0}(T)}}
$$

where $\rho_{x x}$ is measured in units of its critical value, $\rho_{x x c}$ (=29.6 k $\Omega$ for this sample), a normalization which we adopt throughout this letter, and $\nu_{0}(T)$ is a $T$-dependent logarithmic slope, introduced here for the first time. Eq. 2 leads to the first new result of this work: Evidently, data described by it has the necessary symmetry required by Eq. 11. The range over which Eq. 2 holds is quite extensive: As can be seen in Fig. 1, Eq. 2 is a good description of our data for more than 4 orders of magnitude in $\rho_{x x}$ with only small, non-systematic, deviations over a large range of $\nu$. At lower $\nu$ 's systematic deviations appear due to nonohmic effects in the insulator while in the QH phase our measurement is effectively limited to $\nu<0.8$ where $\rho_{x x}$ becomes too small to detect at low $T$. Therefore, we can only put a lower bound on the $\nu$-range of the applicability of Eq. 2.

So far, we demonstrated that $\rho_{x x}$ data near the $\mathrm{QH}-$ insulator transition can be described by a single, welldefined, expression that holds on both sides of the transition. This is potentially useful from the prevalent theoretical standpoint, which asserts that transport coefficients such as $\rho_{x x}$ should be described, near the transition, by a scaling form 15, 16]:

$$
\rho_{x x}=\rho_{x x c} f\left(\frac{\Delta \nu}{T^{1 / z \nu}}\right)
$$

where $f(X)$ is a universal function, and $\nu, z$ and $\rho_{x x c}$ are 
the critical exponents and amplitude of the transition, respectively, and are also expected to be universal [17] $(\nu$ here should not be confused with the LL filling factor).

It is therefore natural to try and associate the scaling function, $f(X)$, with the experimentally derived Eq. 2. This could lead to a determination of the product of the scaling exponents, $z \nu$, which can then be compared with theoretical estimates 15. We will next show that rather surprisingly, upon trying this association using the experimental data, we are faced with a contradiction that leads us to conclude that our data cannot be described by a scaling form. Instead, a form of behavior new to twodimensional electron systems (2DES) at high $B$ emerges, for which no theoretical understanding yet exists.

Inspecting Eqs. 2 and 3 shows that the simplest way of comparing them is by fitting the $\nu_{0}(T)$ data to $T^{1 / z \nu}$. In fig. $2 \mathrm{a}$ we attempt this comparison by plotting the $\nu_{0}$ values obtained from the sample of Fig. 1 vs. their T's, using a log-log graph. Clearly, the fit (dashed line, with the optimal value of $1 / z \nu=0.64$ ) fails at low $T$, and other attempts using a modified $1 / z \nu$ power are equally unsuccessful, as they should be: The data significantly deviates from a power-law behavior expected from the scaling prediction (a straight line on a log-log plot).

An alternative view is revealed in Fig. $2 b$, where the data are plotted on a linear scale: They are suggestive of a linear dependence,

$$
\nu_{0}(T)=\alpha T+\beta,
$$

with $\alpha=0.088 \mathrm{~K}^{-1}$ and $\beta=0.0537$ for this sample. It is the offset, $\beta$, rather than the linear dependence itself, which renders the scaling description and its associated power-law $T$ dependence unsuitable to describe our data. (Even if one does not accept the linear description of the data it is still clear, unless a different conduction mechanism takes over at still lower $T$ 's, that $\nu_{0}(T=0) \neq 0$ and the transition is of finite width.) We emphasize that this linear dependence, with $\beta>0$, is a very general result seen in the 20 samples studied, which are made from various semiconductor materials and fabrication techniques. The parameters $\alpha$ and $\beta$ are sample-dependent, with $\alpha=0.034-0.24 \mathrm{~K}^{-1}$ and $\beta=0.003-0.054$. We found that their variations are correlated and $\beta / \alpha$, which defines a new $T$ scale for the conduction process, seems to be close to $0.5 \mathrm{~K}$ for InGaAs/InP samples and $50 \mathrm{mK}$ for GaAs/AlGaAs samples. The physical significance of this temperature scale is yet unclear.

Putting Eqs. 2 and 1 together leads to our final phenomenological form:

$$
\rho_{x x}=e^{\left(\frac{-\Delta \nu}{\alpha T+\beta}\right)} .
$$

This compact expression describes the $\rho_{x x}$ data over a very large range of both $T$ and $\nu$, and holds for all our samples.
Since our failure to 'scale' our data in the usual way is a direct result of the finite $\beta$, it becomes imperative to establish whether this observation is an integral part of the transition, or an experimental artifact, with $\beta$ vanishing in the appropriate limits of infinite sample size and zero excitation current. Naturally, a definite answer to any of these questions is, at best, hard to obtain. Nevertheless, we would like to present several compelling arguments that alleviate some of these concerns. First, all our samples are relatively large, ranging from $100 \times 300 \mu \mathrm{m}$ to $1 \times 1 \mathrm{~mm}$ in size. Even at $\mathrm{mK}$ temperatures, it is not expected that such samples will exhibit finite-size effects. Second, we found that our results, namely the parameters presented in Table 1, are independent (within error) of excitation currents that are as much as 10 times larger than the currents used in this study $(0.01-1 \mathrm{nA})$. Thus, non-ohmic heating effects are unlikely to be the cause of the finite $\beta$. Increasing the current further resulted in significant deviation from the behavior described by Eq. 2. Third, the results presented in this letter are quite general to all our samples, which rules out the possible that gross sample inhomogeneities and imperfections (on a scale comparable to the sample size) dominate the transport. Smaller scale inhomogeneities and disorder are certain to play a role, which is not yet understood.

The next logical step in light of the simple and general form $\rho_{x x}$ takes is to consider the behavior of the Hall resistivity, $\rho_{x y}$. Unfortunately at this stage a clear experimental answer can not be given, due to the lack of consistent results. For some of our samples we have, however, obtained a $\rho_{x y}$ value that remains quantized beyond the QH state into the insulating phase [10]. Incidentally, given the symmetry of $\rho_{x x}$, a $\rho_{x y}$ which is indeed quantized on both sides of the transition is a prerequisite for a symmetric $\sigma_{x x}$.

The inconsistency of our data with a scaling description is troubling if we recall several earlier results that were taken to indicate the proximity of the transition region to a quantum critical point [16]. While two of these results, namely the existence of a universal (within 25\%) critical resistivity at the transition [7, 18] and the observation of a reflection symmetry consistent with charge-flux duality [9] are consistent with the data presented here, the indication of scaling behavior observed by Wei et al. [1] is clearly not. This is particularly puzzling since some of our measurements were done on InGaAs/InP samples that are from the same growth as the sample in Ref. 四. We can not rule out a scenario in which the sample of Ref. [1] is of exceptional homogeneity and is therefore a better representative of the ideal theoretical case. It is conceivable that our samples would crossover to similar scaling behavior at (yet inaccessible) lower T's. We may remark, however, that a universal critical amplitude was not observed for the transitions studied by Wei et al., which is actually in conflict with the scaling framework. We should also note that our previous scaling anal- 
yses of the QH-insulator transition 19], which attempted to "collapse" a narrow range of $\rho_{x x}(\nu, T)$ data near the transition, were fairly successful despite the fact that the data follows Eq. 5 with $\beta>0$. Attempts to collapse numerical "data" generated using Eq. 5 with parameters obtained from our samples acutely demonstrate that using a limited range of data can give false indication of quantum-critical behavior. Needless to say, they also call into question our previous belief that the symmetries of the transport reflect symmetries of an underlying quantum-critical point.

In this letter we argued that the $\nu$ range where Eq. 5 holds is not a critical region of a quantum phase transition. It is important to note that this regime is distinct from a fully developed QH liquid or an insulator as well, for it extrapolates, through the positive-definite $\beta$, to a finite resistivity at $T=0$ for $\nu$ both bigger and smaller than $\nu_{c}$. Whether this new regime of 2DES at high- $B$ can be sharply defined is unclear. But even if it were to give way to one, or more, of the familiar phases of $2 \mathrm{DES}$ in the ideal limit of vanishing $T$ 's and highly homogeneous material, the extremely wide range of applicability of Eq. 5 both in $T$ and $\nu$, over such a diversity of samples, coupled with its introduction of a new, $B$-dependent temperature scale, $\beta / \alpha$, to the conduction process render this new regime a very important, as well as interesting, topic of research.

[1] H. P. Wei, D. C. Tsui, M.A. Paalanen and A. M. M. Pruisken, Phys. Rev. Lett. 61, 1294 (1988).

[2] S. Koch, R. J. Haug, K. v. Klitzing and K. Ploog, Phys. Rev. Lett. 67, 883 (1991).

[3] H. W. Jiang, C. E. Johnson, K. L. Wang, and S. T. Hannahs, Phys. Rev. Lett. 71, 1439 (1993).

[4] T. Wang, K. P. Clark, G. F. Spencer, A. M. Mack, and W. P. Kirk, Phys. Rev. Lett. 72, 709 (1994).

[5] R. J. F. Hughes, J. T. Nicholls, J. E. F. Frost, E. H. Linfield, M. Pepper, C. J. B. Ford, D. A. Ritchie, G. A. C. Jones, E. Kogan, and M. Kaveh, J. Phys. Condens. Matter 6, 4763 (1994).

[6] B. W. Alphenaar and D. A. Williams, Phys. Rev. B 50, 5795 (1994).

[7] D. Shahar, D. C. Tsui, M. Shayegan, R. N. Bhatt and J. E. Cunningham, Phys. Rev. Lett. 74, 4511 (1995).

[8] T. Okamoto, Y. Shinohara and S. Kawaji, Phys. Rev. B 5211109 (1995).

[9] D. Shahar and D. C. Tsui and M. Shayegan and E. Shimshoni and S. L. Sondhi, Science 274, 589 (1996).

[10] D. Shahar, D. C. Tsui, M. Shayegan, J. E. Cunningham, E. Shimshoni and S. L. Sondhi, Solid State Comm. 102, 817 (1997).

[11] J. K. Jain, Phys. Rev. Lett. 63, 199 (1989).

[12] E. Shimshoni, S. L. Sondhi and D. Shahar, Phys. Rev. B (in press).

[13] D. Simonian, S. V. Kravchenko and M. P. Sarachik, Phys. Rev. B 55, R13421 (1997).

[14] V. Dobrosavljevic, E. Abrahams, E. Miranda and S. Chakravarty, Phys. Rev. Lett. (submitted).

[15] B. Huckestien, Rev. Mod. Phys. 67, 357 (1995).

[16] S. L. Sondhi, S. M. Girvin, J. P. Carini and D. Shahar, Rev. Mod. Phys. 69, 315 (1997);

[17] S. A. Kivelson, D. H. Lee, and S. C. Zhang, Phys. Rev. B 46, 2223 (1992).

[18] L. W. Wong, H. W. Jiang, N. Trivedi and E. Palm, Phys. Rev. B 51, 18033 (1995).

[19] W. Pan, D. Shahar, D. C. Tsui, H. P. Wei and M. Razeghi, Phys. Rev. B (in press).

Correspondence and requests for materials should be addressed to D.S. (e-mail: dannys@princeton.edu).

FIG. $1 . \rho_{x x}$ vs. $\nu$ at $T=72,293,518,940,1461$ and 2210 $\mathrm{mK}$, for an InGaAs/InP sample, RA609C. Inset: $\rho_{x x}$ (linear scale) vs. $\nu$ at $T=293,940,1461$ and $2210 \mathrm{mK}$. The arrow indicates the transition at $\nu_{c}=0.562$, which is the common crossing point of the $\rho_{x x}$ isotherms.

FIG. 2. (a) A log-log graph of $\nu_{0}$ of Eq. 1 vs $T$ obtained from a fit of the $\rho_{x x}$ traces of sample RA609C (Fig. 1) to Eq. 2 (b) Same as (a), plotted using a linear graph. Solid lines are least-square fit to Eq. 4 resulting in $\alpha=0.0878 \mathrm{~K}^{-1}$ and $\beta=0.05367$. Dashed line in (a) is the optimal power-law fit: $\nu_{0} \sim T^{1 / z \nu}$, with $1 / z \nu=0.64$. Inset: Same as (b), for a GaAs/AlGaAs sample, with the linear fit (solid line) resulting in $\alpha=0.24 \mathrm{~K}^{-1}$ and $\beta=0.014$. 


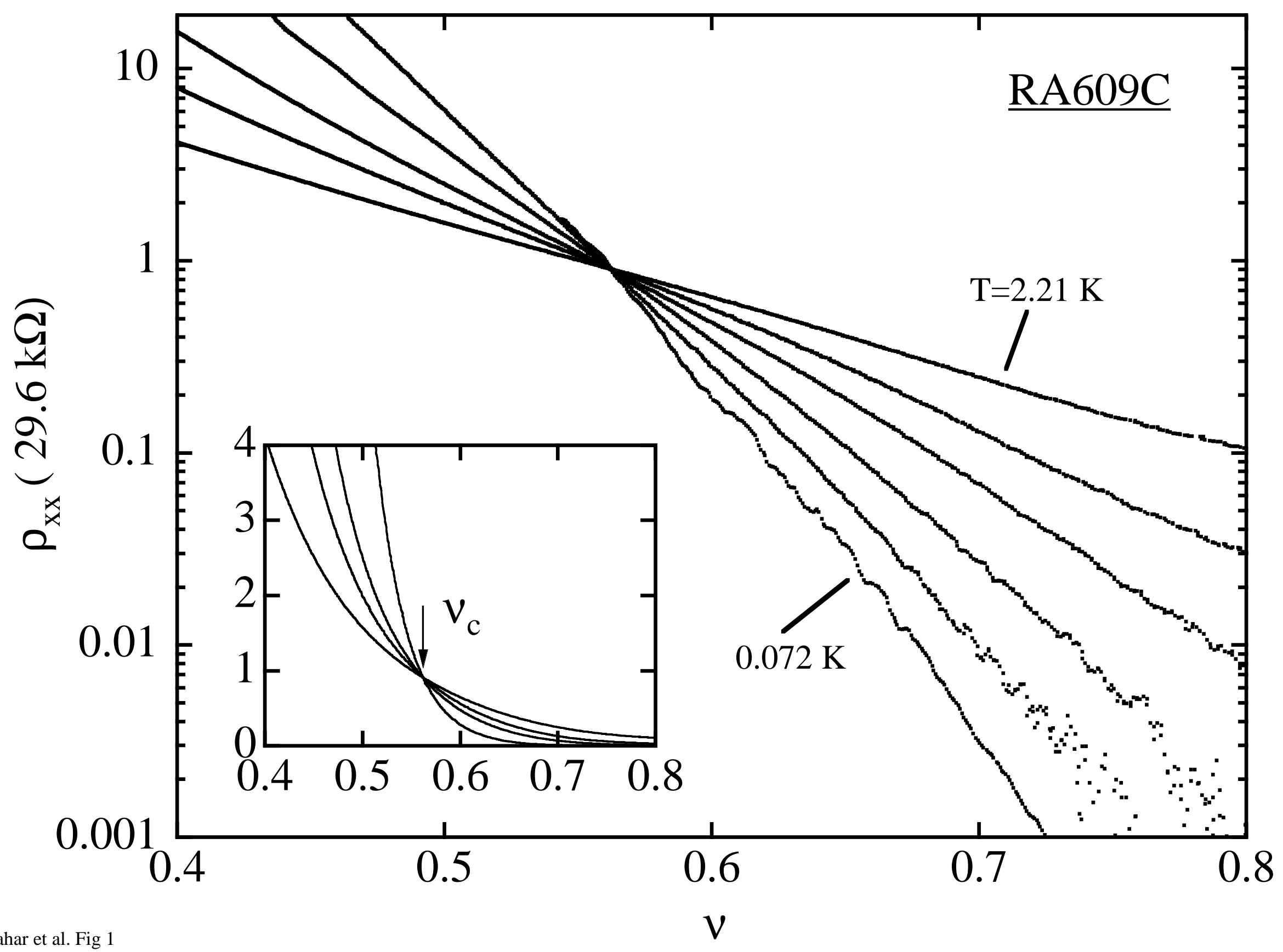



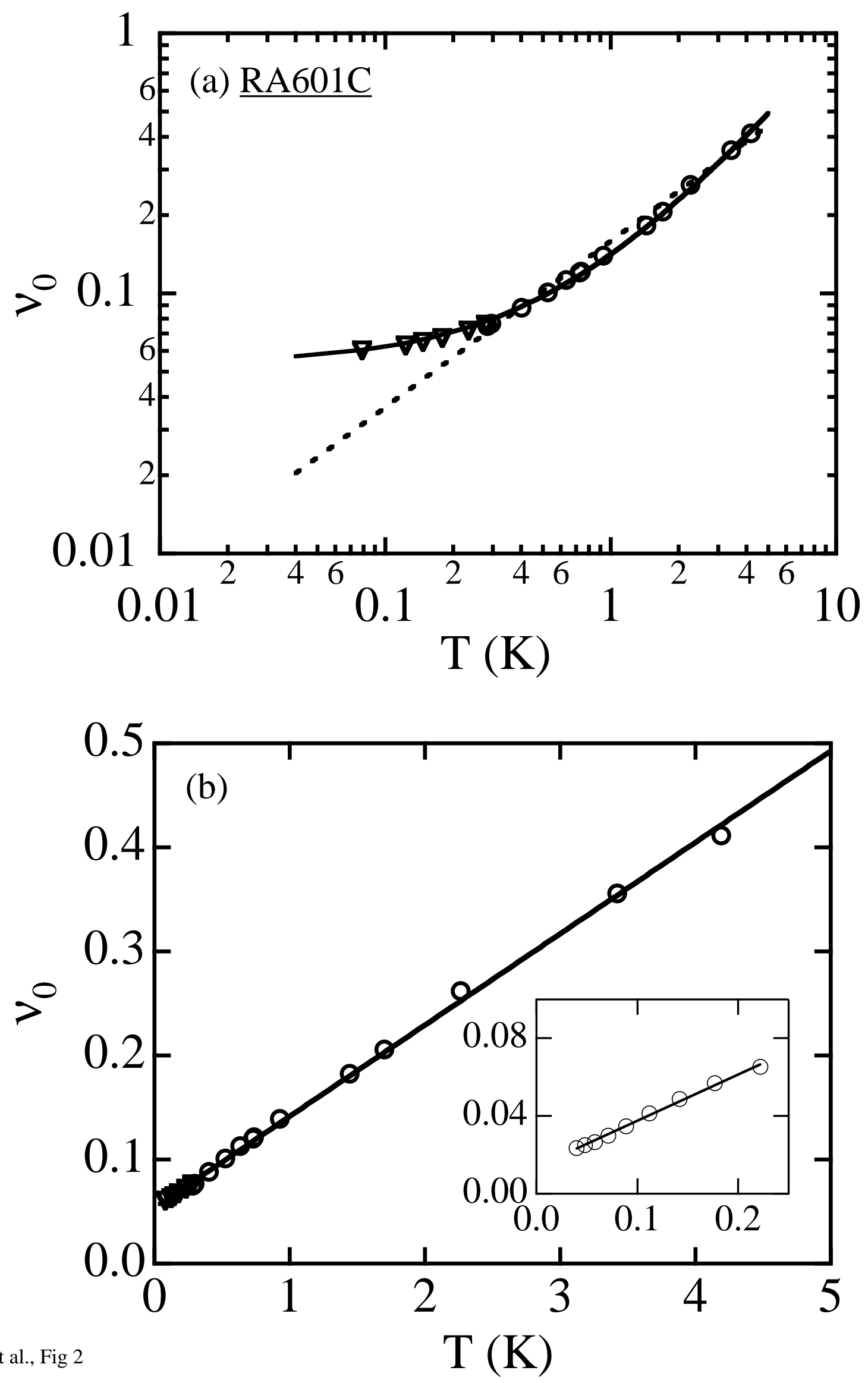

D. Shahar et al., Fig 2 